\title{
Family of Flexible Multivariate Distributions with Applications in Empirical Finance
}

\author{
B. MAZUR* AND M. PiPIEŃ \\ Cracow University of Economics, Rakowicka 27, PL 31517 Kraków \\ Doi: 10.12693/APhysPolA.138.65 \\ *e-mail: blazej.mazur@uek.krakow.pI
}

\begin{abstract}
We develop a class of parametric distributions that are capable of accounting for non-standard empirical properties that are evident in some financial time series. We aim at creating a parametric framework that allows for serious divergences from the multivariate Gaussian case both in terms of properties of marginal distributions and in terms of the dependence pattern. We are particularly interested in obtaining a multivariate construct that allows for considerable degree of heterogeneity in marginal properties of its components (like tail thickness and asymmetry). Moreover, we consider non-standard dependence patterns that go beyond a linear correlation-type relationship while maintaining simplicity, obtained by introducing rotations. We make use of marginal distributions that belong to generalized asymmetric Student- $t$ class analysed in [A. Harvey, R.J. Lange, J. Time Ser. Anal. 38, 175 (2017)], allowing not only for skewness but also for asymmetric tail thickness. We illustrate flexibility of the resulting bivariate distribution and investigate its empirical performance examining unconditional properties of bivariate daily financial series representing dynamics of stock price indices and the related FUTURES contracts, as well as analysing unconditional co-dependence between daily returns on DAX and FTSE indices.
\end{abstract}

topics: Bayesian inference, generalized asymmetric $t$-distribution, skewness, orthogonal matrices, rotations

\section{Introduction}

The empirical distributions of economic variables might display serious divergences from the multivariate normal (Gaussian) case, see [1]. The introduction of the probability distribution that would depart from the Gaussian case occurred for the first time more than 130 years ago [2-6]. During last four decades this journey beyond normality has been particularly desired for a proper modelling the empirical distribution of data that characterise fluctuations observed on financial markets. However, the majority of theoretical investigations in the field of finance that have been developed over last half a century rely on the fundamental assumption that the distribution for related financial returns is Gaussian. The well known example of the explicit assumption of normality is the framework that enables to price an option, developed in $[7,8]$. In other areas of finance, the Gaussian assumption is made implicitly. For example, the theory of mean-variance portfolio selection derived from [9] does not require normality of returns, but does assume that distribution of asset returns are characterised by their first and second moment. Consequently, the utility function taken into account by a decision maker is a function only of the mean and variance of a portfolio returns. Implicit normality is also rooted in the capital asset pricing model (CAPM) elaborated on the basis of Markovitz' efficient frontier.

On the other hand, with rare exceptions, empirical analyses that have been performed since 1960 's fail to find any support for the normality axiom. In the 1990's, the joint effort of economists and physicists resulted with mathematical models that provided support for return distributions with heavy tails. In particular, the departure from the Gaussian distribution of financial returns was justified by modelling the interactions of market agents [9-13].

The feature being at least as important as heavy tails is skewness (or asymmetry). Some serious attempts to model it for the multivariate case have been made during last decades. Some earlier studies conducted in the univariate case [14] were motivated by the statement that unmodelled skewness may have a serious impact on inference on other parameters in the model. They illustrated this effect discussing the role of skewness in modelling the relationship between risk and return. Recently more extended discussion on this topic are presented $[15,16]$. The influence of skewness of the distribution of asset returns was analysed in the problem of portfolio selection [17] and also in $[18,19]$. Recently, the impact of heavy tails and asymmetry on the results of the event study analysis were performed in [20]. Theodossiou [21] presented 
empirical importance of the Skewed GED family of distributions of financial assets in the problem of option pricing, while in [22] use the family (with dynamic skewness) to model differences between upside and downside shocks in series of freight rates.

Motivating the importance of asset pricing model that incorporates distributional asymmetry it was emphasized [19] that systematic skewness is economically important and governs risk premium. Adcock [24] relaxed assumption of the multivariate Gaussian distribution in the CAPM model and presented results indicating empirical importance of the family of multivariate Skewed-Normal distributions (see [25]) for UK Stocks.

Also many authors tried to go beyond conditional normality assumed commonly in case of Multivariate GARCH (M-GARCH) models. For example, conditionally elliptical distribution in DCC model was presented in [26]. Some other non-Gaussian conditional distributions were analysed in [27-29]. However, commonly applied econometric strategy using the Maximum Likelihood estimation procedure might result with a considerable small-sample bias [30].

There is no doubt that proper modelling of empirical features observed for the case of related financial time series requires construction of a flexible class of distributions. Moreover, the development of alternative methods of statistical inference is necessary. In this paper we address these two issues. We propose a novel class of parametric bivariate distributions to model empirical properties that are evident in some financial time series. We depart from the multivariate Gaussian case both in terms of properties of the marginal distributions and in terms of the co-dependence pattern. In order to achieve flexibility we make use of marginal distributions that belong to generalized asymmetric Student- $t$ class analysed by Harvey and Lange [31], allowing not only for skewness but also for asymmetric tail thickness. We also develop methods of formal Bayesian inference and present posterior analysis within constructed class of sampling models. We also consider the issue of Bayesian (density) prediction. In the paper we illustrate flexibility of the resulting bivariate distribution and investigate its empirical performance examining unconditional properties of bivariate daily financial series representing dynamics of stock price indices and the related FUTURES contracts.

All results were obtained on the basis of original numerical procedures developed within Oxmetrics environment.

\section{A family of non-standard bivariate distributions}

To start let us consider $n$-variate random variable $z_{[n x 1]}=\left(z_{1}, \ldots, z_{n}\right)^{\prime}$ with independent coordinates, i.e., with $p(z)=\prod_{i=1}^{n} p_{i}\left(z_{i}\right)$. The shape of isodensities of bivariate $z$ is presented in Fig. 1 .
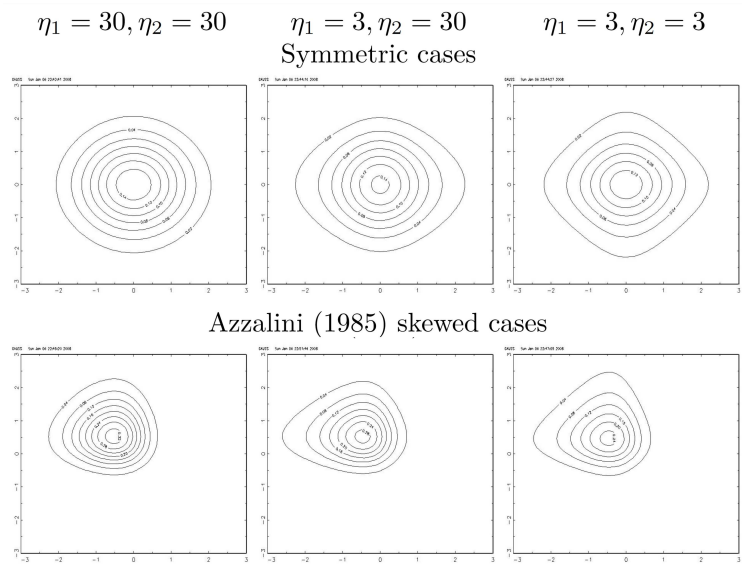

Fig. 1. Plots of isodensities of bivariate distribution with independent Student- $t$ (first row) or skewed Student- $t$ (second row) coordinates with $\eta_{i}, i=1,2$ degrees of freedom parameters.

To define $p_{i}\left(z_{i}\right)$ we utilize Student- $t$ univariate distributions with barely Gaussian tails (degrees of freedom parameter $\eta_{i}=30$ ) or much heavier ones $\left(\eta_{i}=3\right)$. We also analyse the effect of imposing hidden truncations mechanism [32] resulting with a skewed Student- $t$ distributions. Analysing plots in Fig. 1, it is clear that possible outliers and asymmetry can be captured by distribution $p(z)$ only if these features of the data will occur along original coordinate axes, defined by canonical basis in $R^{n}$. Also any family of distributions $p(z)$ is not closed with respect to the orthogonal transformation of the components $z_{i}, i=1, \ldots, n$, i.e., is not coordinate free [33].

In the next step one may consider a distribution resulting from a linear transformation:

$$
\varepsilon=A z+b .
$$

For a nonsingular matrix $A_{[n x n]}$ and $b \in R^{n}$ the distribution of $\varepsilon$ is described by a well-defined density of the following form:

$$
p(\varepsilon)=\frac{1}{|\operatorname{det}[A]|} \prod_{i=1}^{n} p_{i}\left(A^{-1(i)}(\varepsilon-b)\right),
$$

where $A^{-1(i)}$ denotes $i$-th row of matrix $A^{-1}$. In what follows make use of this result in a bivariate setting, going far beyond the standard scheme where $A$ is defined as a root of symmetric and positive definite matrix generating the covariance structure.

To define the univariate distributions $p_{i}$ we apply the generalised Student $t$-distribution proposed recently by [31]. It generalizes previous results $[34,35]$, as well as $[36,37]$ among others, see the references and discussion in [31].

However, the form used here is re-scaled to ensure unit variance. The resulting probability density function (with mode at 0 and nonzero mean in general) is 


$$
\begin{aligned}
& f_{\mathrm{GAST}}\left(z ; h, \alpha, v_{\mathrm{L}}, v_{\mathrm{R}}, \eta_{\mathrm{L}}, \eta_{\mathrm{R}}\right)=\frac{K_{\mathrm{LR}} \sqrt{f}}{\sqrt{h}} \\
& \quad \times\left\{\begin{array}{l}
\left(1+\frac{1}{\eta_{\mathrm{L}}}\left(\frac{-z \sqrt{f}}{2 \alpha \sqrt{h}}\right)^{v_{\mathrm{L}}}\right)^{-\frac{1+\eta_{\mathrm{L}}}{v_{\mathrm{L}}},}, z \leq 0 \\
\left(1+\frac{1}{\eta_{\mathrm{R}}}\left(\frac{z \sqrt{f}}{2(1-\alpha) \sqrt{h}}\right)^{v_{\mathrm{R}}}\right)^{-\frac{1+\eta_{\mathrm{R}}}{v_{\mathrm{R}}}}, z>0
\end{array}\right.
\end{aligned}
$$

where GAST stands for "generalized asymmetric skew $t$ " and $h$ denotes variance. The distribution is a two-piece version of a generalized $t$ distribution, parameter $0<\alpha<1$ introduces skewness, with $\alpha=0.5$ denoting the absence of skewing (symmetry requires also $\eta_{\mathrm{L}}=\eta_{\mathrm{R}}$ and $\left.v_{\mathrm{L}}=\eta_{\mathrm{R}}\right)$, v's control shape around the mode (being more flat or spiked, in a GED-like manner, with $v=2$ leading to Student- $t$ type shape), while $\eta$ 's affect tail thickness (we require that $\eta_{\mathrm{L}}, \eta_{\mathrm{R}}>2$ to ensure that variance is finite). However, the influence of $\eta$ 's and $v$ 's on tail behaviour is not separated clearly. Setting $v_{\mathrm{L}}=v_{\mathrm{R}}=2$ and $\eta_{\mathrm{L}}=\eta_{\mathrm{R}}$ leads to Skewed Student- $t$ case, with Skew Normal and Normal distributions being the limiting ones with $\eta_{\mathrm{L}}$ and $\eta_{\mathrm{R}} \rightarrow \infty$. Hence, the asymmetric and flexible distribution encompasses a number of well-known distributions, including the GED (Generalised Error Distribution) family $\left(\eta_{\mathrm{L}}\right.$ and $\left.\eta_{\mathrm{R}} \rightarrow \infty\right)$. We assume $\eta_{\mathrm{L}}, \eta_{\mathrm{R}}>2$ and $v_{\mathrm{L}}, v_{\mathrm{R}}>1$. In fact, $K_{\mathrm{LR}}$ and $f$ denote rather complicated functions of shape parameters (details of $K_{\mathrm{LR}}$ and $\alpha^{*}$ are given in [31]), note that $P\{z<0\}=\alpha^{*}$, and $f=d-c^{2}$ with

$$
\begin{aligned}
& c=-2 \alpha^{*} \alpha \frac{\eta_{\mathrm{L}}^{\frac{1}{v_{\mathrm{L}}}} \Gamma\left(\frac{2}{v_{\mathrm{L}}}\right) \Gamma\left(\frac{\eta_{\mathrm{L}}-1}{v_{\mathrm{L}}}\right)}{\Gamma\left(\frac{\eta_{\mathrm{L}}}{v_{\mathrm{L}}}\right) \Gamma\left(\frac{1}{v_{\mathrm{L}}}\right)} \\
&+2\left(1-\alpha^{*}\right)(1-\alpha) \frac{\eta_{\mathrm{R}}^{\frac{1}{v_{\mathrm{R}}}} \Gamma\left(\frac{2}{v_{\mathrm{R}}}\right) \Gamma\left(\frac{\eta_{\mathrm{R}}-1}{v_{\mathrm{R}}}\right)}{\Gamma\left(\frac{\eta_{\mathrm{R}}}{v_{\mathrm{R}}}\right) \Gamma\left(\frac{1}{v_{\mathrm{R}}}\right)}, \\
& d=-4 \alpha^{*} \alpha^{2} \frac{\eta_{\mathrm{L}}^{\frac{2}{v_{\mathrm{L}}}} \Gamma\left(\frac{3}{v_{\mathrm{L}}}\right) \Gamma\left(\frac{\eta_{\mathrm{L}}-2}{v_{\mathrm{L}}}\right)}{\Gamma\left(\frac{\eta_{\mathrm{L}}}{v_{\mathrm{L}}}\right) \Gamma\left(\frac{1}{v_{\mathrm{L}}}\right)} \\
&+4\left(1-\alpha^{*}\right)(1-\alpha)^{2} \frac{\eta_{\mathrm{R}}^{\frac{2}{v_{\mathrm{R}}}} \Gamma\left(\frac{3}{v_{\mathrm{R}}}\right) \Gamma\left(\frac{\eta_{\mathrm{R}}-2}{v_{\mathrm{R}}}\right)}{\Gamma\left(\frac{\eta_{\mathrm{R}}}{v_{\mathrm{R}}}\right) \Gamma\left(\frac{1}{v_{\mathrm{R}}}\right)} .
\end{aligned}
$$

Consider a product measure-type bivariate generalised Student- $t$ distribution of the form:

$$
p(z)=p\left(z_{1}, z_{2}\right)=p_{Z_{1}}\left(z_{1}\right) p_{Z_{2}}\left(z_{2}\right),
$$

where $p_{Z_{i}}\left(z_{i}\right)$ is the generalised Student- $t$ distribution of [18] with individual shape parameters, transformed into the above GAST form (parameterised in terms of variance). The only restriction is that each coordinate in $z$ has the same variance $h_{1}$, i.e., $V(z)=h_{1} I$. The existence of variance could in principle be relaxed (in a scale-driven model) in order to allow for e.g. Cauchy-type tails. Now assume that the variable $z$ is subject to a linear

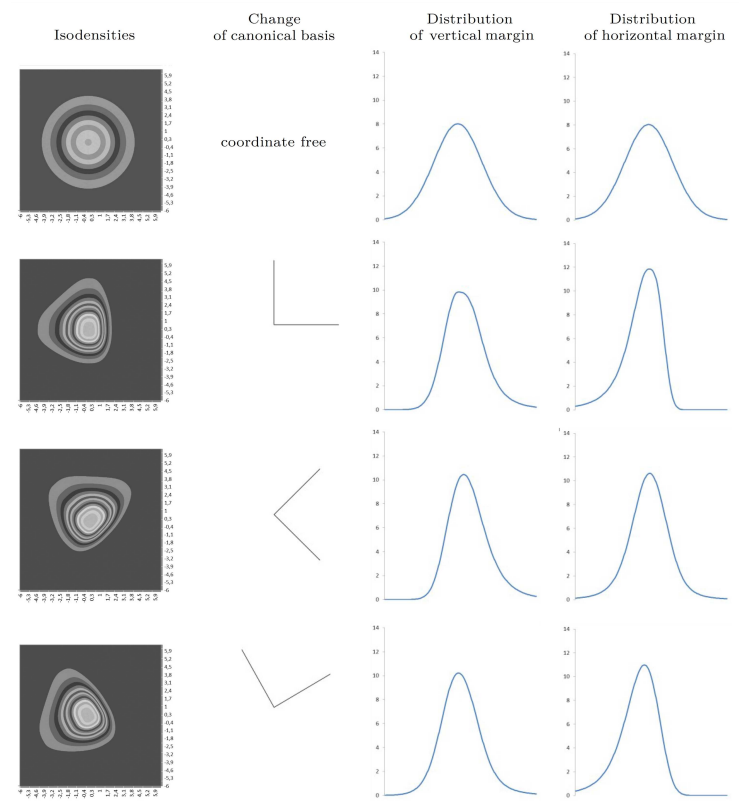

Fig. 2. Plots of isodensities, transformation of the canonical basis and marginal distributions of coordinates in Gaussian case (first row) as well as in case of $v=R_{(\varphi)} z$, for $\varphi=0$ (second row), $\varphi=\frac{\pi}{4}$ (third row), $\varphi=-\frac{\pi}{6}$ (fourth row).

transformation, but the transformation matrix is orthogonal, i.e.: $v=R_{(\varphi)} z$, where:

$$
R_{(\varphi)}=\left[\begin{array}{cc}
\cos (\varphi) & \sin (\varphi) \\
-\sin (\varphi) & \cos (\varphi)
\end{array}\right]
$$

The matrix $R_{(\varphi)}$ imposes clockwise rotation by angle $\varphi, R_{(\varphi)}^{-1}=R_{(\varphi)}^{\prime}$ with $\operatorname{det}\left(R_{(\varphi)}\right)=1$, the transformation might affect the density type but leaves the covariance structure intact. Thus, $v_{i}$ 's are uncorrelated with the same variances, but their marginal distribution might change (relative to those of $z_{i}^{\prime}$ 's). The density of the distribution of $v$ is given by the formula:

$$
\begin{aligned}
& p(v)=\operatorname{det}\left(R_{(\varphi)}\right) p_{Z_{1}}\left(R_{(\varphi)}^{(1)^{\prime}} v\right) p_{Z_{2}}\left(R_{(\varphi)^{\prime}}^{(2)^{\prime}} v\right)= \\
& p_{Z_{1}}\left(R_{(\varphi)}^{(1)^{\prime}} v\right) p_{Z_{2}}\left(R_{(\varphi)}^{(2)^{\prime}} v\right) .
\end{aligned}
$$

Figure 2 shows how the shape of the isodensities of $v$ varies with respect to different values of the shape and the asymmetry parameters. In each case we analyse distributions with variances for margins equal to 4 . In the first row we plotted the reference case as the bivariate Gaussian distribution, being a limiting case here. Possible directional asymmetry and different tail behaviour is presented by the isodensities in the second row. The effect of rotation by a different angle is shown in the third and fourth row. The most interesting property of the analysed distributions is that the dependence pattern assumes zero correlations.

The (rotated) vector $v$ is subject to a further linear transformation that imposes location (mode) $m$ 


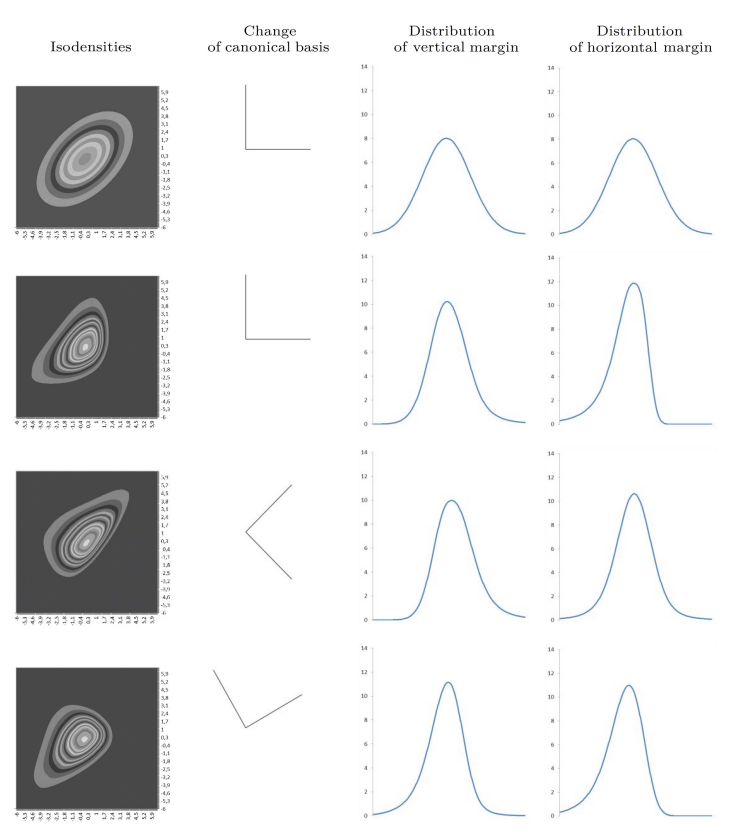

Fig. 3. Plots of isodensities, transformation of the canonical basis and marginal distributions of coordinates in Gaussian case (first row) as well as in case of $y=C v$, for $\varphi=0$ (second row), $\varphi=\frac{\pi}{4}$ (third row), $\varphi=-\frac{\pi}{6}$ (fourth row). In each case we keep the same variances for margins equal to 4 and correlation $\rho=0.5$.

and covariance structure (i.e., correlation and differences in variances) upon $v$ :

$$
y=C v+m .
$$

The matrix $C$ can be parametrized using different concepts of matrix roots, though here we assume that it has the following form:

$$
C=\left[\begin{array}{cc}
\sqrt{1-\rho^{2}} & \rho \\
0 & \sqrt{\frac{h_{2}}{h_{1}}}
\end{array}\right] .
$$

Then $h_{i}$ are variances of coordinates and $\rho \in(-1,1)$ represents the correlation coefficient between $y_{1}$ and $y_{2}$. The density of the distribution of $y$ is given by the following formula:

$$
\begin{aligned}
& p(y)=\operatorname{det}\left[C^{-1}\right] p_{Z_{1}}\left(R_{(\varphi)}^{(1)^{\prime}} C^{-1}(y-m)\right) \\
& \quad \times p_{Z_{2}}\left(R_{(\varphi)}^{(2)^{\prime}} C^{-1}(y-m)\right) .
\end{aligned}
$$

Figure 3 depicts isodensities of some exemplary cases of distribution of $y=C v$. We analyse correlated versions of distributions presented in Fig. 2. In each case we assumed correlation coefficient $\rho=0.5$. Bivariate distributions presented in Fig. 3 show remarkable degree of flexibility in modelling structure of observables, though it does not exceed the case of a linear transformation of the product measure initially defined for a vector $p(z)$. Its flexibility results from the fact that all the shape parameters could be made dimension-specific (in the space of $z$ 's). Crucially, the original formulation in [29] allows for a complicated asymmetry pattern which is here generalized to a higher dimension. The distribution is unimodal by construction (which is not necessarily true about some other flexible constructs like mixtures), however its mean is a complicated function of all the model parameters. The rotation angle $\varphi$ is identified if $p(z)$ defines a distribution class that is not closed under rotations, which holds almost everywhere in the parameter space considered here. However, e.g. for a (limiting) special case of bivariate Gaussian distribution, $\varphi$ would be locally unidentified.

\section{Empirical illustration 1: S\&P500 SPOT and FUTURES}

We analysed daily logarithmic returns of the S\&P500 SPOT and FUTURES together with volumes traded, covering the period from 28.08.2001 till 12.12.2017; 4099 observations. We considered four bivariate datasets, namely the daily returns of the SPOT index with daily returns of the FUTURES volume traded (dataset A), the daily returns of the SPOT index with daily returns of the SPOT volume traded (dataset B), the daily returns of the SPOT index with daily returns of the FUTURES index (dataset $\mathrm{C}$ ) and the daily returns of the SPOT volume traded with the daily returns of the FUTURES volume traded (dataset D). The empirical properties of analysed bivariate series are presented in Fig. 4.

We applied the class of bivariate distributions, presented in the previous part to model the unconditional distribution of analysed series. In order to perform this task we constructed Bayesian models for each analysed series. The estimation was carried over using the Metropolis-Hastings Random Walk sampler; we assume prior independence across all the model parameters. The priors are informative though tailored to convey relatively weak

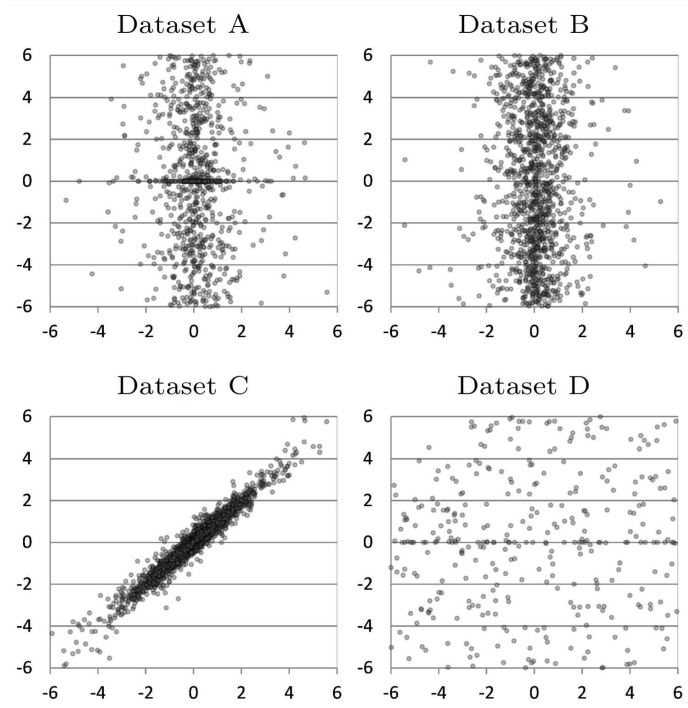

Fig. 4. Analysed bivariate time series (the axes are adjusted to match Fig. 3). 


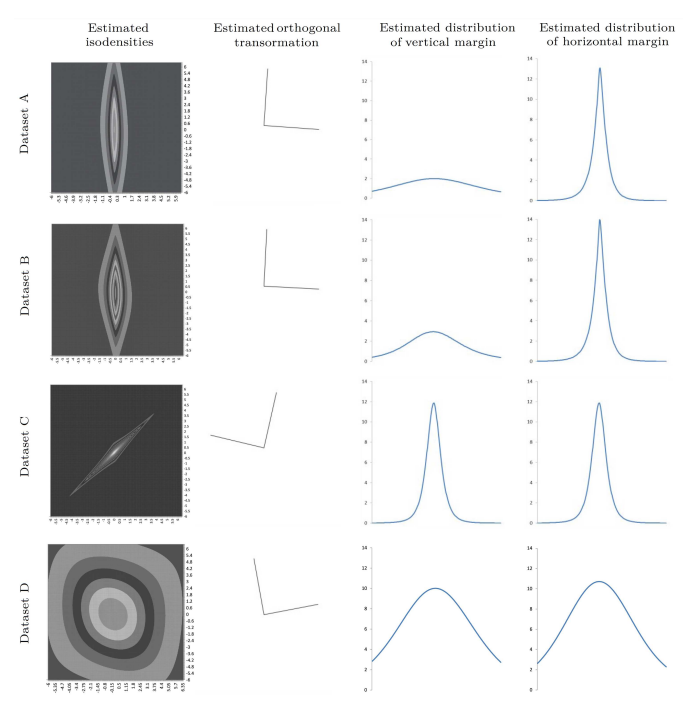

Fig. 5. Estimation results: plots of isodensities and margins for the parameters values corresponding to posterior means.

information, for example for $\varphi, \rho$ and $\alpha$ we assume uniform priors. A posteriori we find limited skewness (with $\alpha=0.5$ being rather likely) but strong shape asymmetry (e.g. clear evidence against $v_{\mathrm{L}}=$ $v_{\mathrm{R}}$ or $\eta_{\mathrm{L}}=\eta_{\mathrm{R}}$ in some cases) which justifies the empirical relevance of shape-asymmetric distributions of $[31,34,35]$. We find support for $v_{\mathrm{L}}<1.5$ (e.g., using SPOT index returns), $2<\eta_{\mathrm{L}}<3$, e.g., using volume growth rates of FUTURES.

The estimated unconditional distributions are presented in Fig. 5. For each dataset we plotted isodensities of the distribution corresponding to $p(y)$ with posterior means of parameters used as plug-in estimates. We report the empirical importance of the rotation effect (relying on the posterior mean of parameter $\varphi$ ). The shapes of resulting marginal univariate distributions are also presented.

In case of datasets $\mathrm{A}$ and $\mathrm{B}$ we see a little data support in favour of dependence. Also the rotation effect seems negligible. The posterior mean of parameter $\varphi$ is equal to 0.068 in case of dataset $\mathrm{A}$ and to 0.050 in case of dataset B. Also both datasets support small negative correlation, indicating no substantial linear dependence between the variables. The posterior mean of the correlation $\rho=-0.089$ in case of dataset A and $\rho=-0.057$ for dataset B.

The strong linear dependence as well as empirical importance of the rotation effect was obtained for the case of dataset C. Estimated posterior mean of parameter $\varphi=-1.345$ indicates strong counter clockwise rotation of coordinates, by more than $75^{\circ}$. The posterior mean of correlation parameter $\rho=0.969$ is rather high in this case. A moderate effect of dependence was obtained in case of dataset D. We report some evidence in favour of the rotation effect, as the posterior mean of $\varphi=-0.185$. It results with counter clockwise rotation of coordinates by about $10^{\circ}$. The dataset $\mathrm{D}$

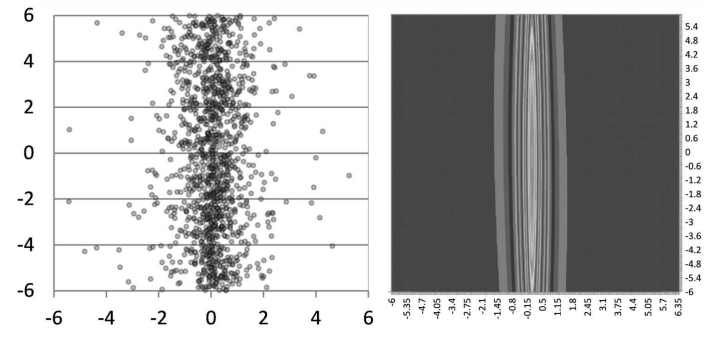

Fig. 6. Dataset B: the data versus the posteriorpredictive density.

can be also described by small positive correlation, since the posterior mean of $\rho=0.236$. The posterior-predictive distribution (that takes into account the estimation uncertainty) is depicted in Fig. 6 (for the dataset B).

\section{Empirical illustration 2: unconditional co-dependence between DAX and FTSE daily returns}

Another case considered here focuses on analysis of joint unconditional distribution of daily returns of FTSE 100 and DAX 30 daily logarithmic returns. The motivation to consider the two series is the following: DAX returns might be perceived as representing a more fundamental perspective of the real side of the EU economy. On the other hand, FTSE represents international financial linkages and corresponds to financial processes. The data cover the period of 03 Jan 1991 till 13 Jul 2017, which (keeping only the trading days for both stock exchange markets) results in 6639 data points. The distribution of analysed series are presented on Fig. 7.

We construct time series of the corresponding returns considering two options. Firstly we define the usual daily returns linking the closing price in a given day with the closing price of the previous working day; the case is labelled SERIES I. Secondly, due to properties of the FTSE index we consider the returns between opening and closing of the same working day (form both indices), which is labelled SERIES II.

For both datasets we estimated unrestricted model (denoted by $M_{\rho, \varphi}$ ) and its two special cases, denoted by $M_{\rho, 0}$ and $M_{0, \varphi}$, obtained by imposing restrictions either $\varphi=0$ (in case of $M_{\rho, 0}$ ) or $\rho=0$ (in case of $M_{0, \varphi}$ ), respectively. The results of posterior inference are presented in Figs. 8-10 and also in Tables I-III. Figure 8 and Table I is related to $M_{\rho, 0}$, Fig. 9 and Table II is related to $M_{0, \varphi}$, while Fig. 10 and Table III contains results obtained in case of unrestricted model $M_{\rho, \varphi}$. In Figs. 8-10 we presented histograms of the marginal posterior distributions of all parameters, while Tables I-III contain posterior summaries (means and standard deviations) of shape parameters of the distribution (2). 
TABLE I

Posterior means and standard deviations of shape parameters and $\rho$ obtained in case of restricted model $M_{\rho, 0}$ with $\varphi=0$ and $\rho \in(-1,1)$.

\begin{tabular}{c|c|c|c|c|c|c|c|c|c|c}
\hline \hline$v_{1 \mathrm{~L}}$ & $v_{2 \mathrm{~L}}$ & $v_{1 \mathrm{R}}$ & $v_{2 \mathrm{R}}$ & $\eta_{1 \mathrm{~L}}$ & $\eta_{2 \mathrm{~L}}$ & $\eta_{1 \mathrm{R}}$ & $\eta_{2 \mathrm{R}}$ & $\varphi$ & $\rho$ \\
\hline \multicolumn{8}{c}{ DATASET I } \\
\hline 1.70 & 1.59 & 1.58 & 2.01 & 6.15 & 4.69 & 6.43 & 3.95 & - & 0.77 \\
0.211 & 0.190 & 0.224 & 0.253 & 1.547 & 1.032 & 2.280 & 0.560 & - & 0.0096 \\
\hline \multicolumn{8}{c}{ DATASET II } \\
\hline 1.36 & 1.66 & 2.027 & 1.84 & 12.06 & 4.33 & 3.88 & 4.03 & - & 0.66 \\
0.183 & 0.199 & 0.303 & 0.232 & 12.107 & 0.815 & 0.559 & 0.632 & - & 0.014
\end{tabular}

TABLE II

Posterior means and standard deviations of shape parameters and $\varphi$ obtained in case of restricted model $M_{0, \varphi}$ with $\rho=0$ and $\varphi \in\left(-\frac{\pi}{2}, \frac{\pi}{2}\right) \approx(-1.57,1.57)$.

\begin{tabular}{c|c|c|c|c|c|c|c|c|c|c}
\hline \hline$v_{1 \mathrm{~L}}$ & $v_{2 \mathrm{~L}}$ & $v_{1 \mathrm{R}}$ & $v_{2 \mathrm{R}}$ & $\eta_{1 \mathrm{~L}}$ & $\eta_{2 \mathrm{~L}}$ & $\eta_{1 \mathrm{R}}$ & $\eta_{2 \mathrm{R}}$ & $\varphi$ & $\rho$ \\
\hline \multicolumn{2}{c}{ DATASET I } & \multicolumn{1}{c}{$c$} \\
\hline 2.27 & 1.20 & 2.71 & 1.59 & 4.41 & 11.45 & 2.03 & 5.70 & 1.04 & - \\
0.268 & 0.114 & 0.313 & 0.177 & 0.583 & 10.636 & 0.006 & 1.437 & 0.090 & - \\
\hline \multicolumn{8}{c}{ DATASET II } \\
\hline 2.23 & 1.06 & 2.71 & 1.18 & 4.32 & 11.73 & 2.01 & 6.18 & 1.37 & - \\
0.252 & 0.040 & 0.315 & 0.100 & 0.579 & 4.203 & 0.0014 & 1.528 & 0.015 & -
\end{tabular}

TABLE III

Posterior means and standard deviations of shape parameters, $\rho$ and $\varphi$ obtained in case of unrestricted model $M_{\rho, \varphi}$, i.e., with $\rho \in(-1,1)$ and $\varphi \in\left(-\frac{\pi}{2}, \frac{\pi}{2}\right) \approx(-1.57,1.57)$

\begin{tabular}{c|c|c|c|c|c|c|c|c|c|c}
\hline \hline$v_{1 \mathrm{~L}}$ & $v_{2 \mathrm{~L}}$ & $v_{1 \mathrm{R}}$ & $v_{2 \mathrm{R}}$ & $\eta_{1 \mathrm{~L}}$ & $\eta_{2 \mathrm{~L}}$ & $\eta_{1 \mathrm{R}}$ & $\eta_{2 \mathrm{R}}$ & $\varphi$ & $\rho$ \\
\hline \multicolumn{1}{c}{ DATASET I } \\
\hline 1.23 & 1.83 & 1.64 & 1.80 & 8.28 & 5.31 & 5.42 & 5.37 & -1.14 & 0.77 \\
0.120 & 0.267 & 0.178 & 0.221 & 3.831 & 1.228 & 1.464 & 1.066 & 0.068 & 0.010 \\
\hline \multicolumn{8}{c}{ DATASET II } \\
\hline 1.06 & 1.96 & 1.25 & 1.98 & 10.79 & 4.19 & 5.50 & 4.51 & -0.69 & 0.67 \\
0.037 & 0.308 & 0.123 & 0.300 & 3.331 & 0.667 & 1.362 & 0.833 & 0.028 & 0.011
\end{tabular}

Analysing results presented in Table III one might note, that in both cases of the data, parameter $\varphi$ has the posterior concentrated away from zero (so is the correlation coefficient $\rho$ ). This indicates substantial empirical evidence in favour of existence of both effects. With reference to posterior analysis of parameter $\varphi \in\left(-\frac{\pi}{2}, \frac{\pi}{2}\right)$ it is clear that both datasets support existence of a difference set of coordinate axes along which shape of distributions of $y_{i}$ 's is modelled by univariate $p_{z_{i}}$ densities of the Harvey and Lange (2017) type in (1). Comparison of posterior inference about $\varphi$ in case of unrestricted model (Table III) with these obtained in case of imposing restrictions of no correlation ( $\rho=0$; model $M_{0, \varphi}$, Table II) one may notice very strong linkage between effect of existence of correlation and orthogonal transformation of coordinates in the mode. Condition to restriction $\rho=0$ posterior density of $\varphi$ changes its distribution, placing the majority of the probability mass in regions of a positive sign of $\varphi$, while in

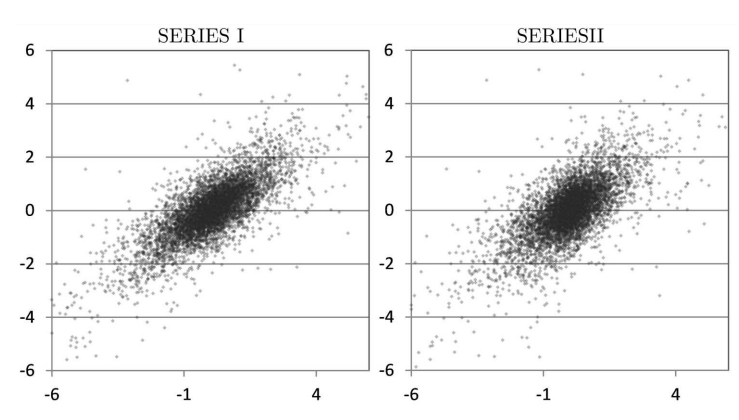

Fig. 7. DAX/FTSE daily returns.

case of unrestricted model the posterior distribution of $\varphi$ indicated positive sign of the angle parameter. Surprisingly we report no opposite linkage, i.e., between orthogonal transformation and correlation. Posterior inference, about parameter $\rho$ remains unchanged in case of unrestricted model (Table III) and the model $M_{\rho, 0}$ with restriction $\varphi=0$, 


\section{DATASET I}

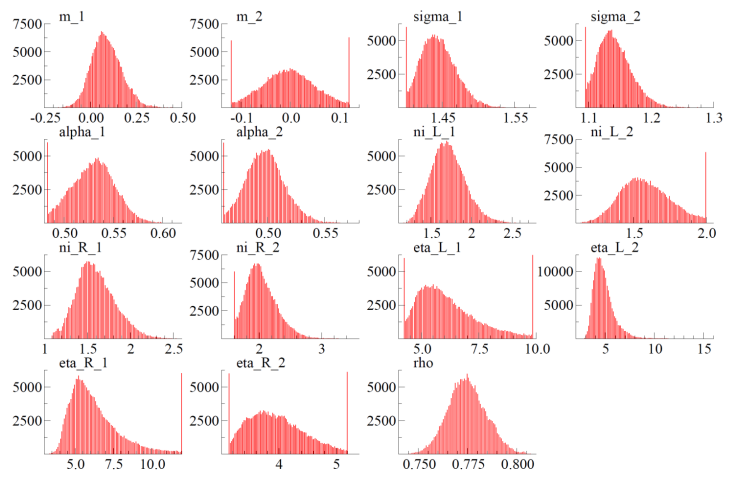

DATASET II

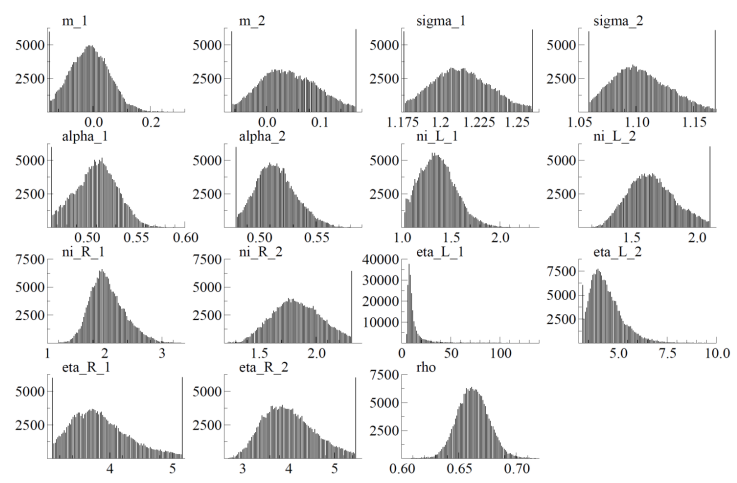

Fig. 8. Histograms of the marginal posterior distributions of parameters obtained in case of restricted model $M_{\rho, 0}$ with $\varphi=0$ and $\rho \in(-1,1)$.

forcing no orthogonal transformation. In both models, $M_{\rho, \varphi}$ and $M_{0, \varphi}$, the data indicate strong unconditional correlation between univariate series, with posterior mean equal to 0.77 for SERIES I and 0.66 for SERIES II.

Consequently, our analysis shows that the effect of correlation is empirically important and the data support non-zero correlation so strongly, that the inference about $\rho$ remains unaffected irrespectively to existence of the orthogonal transformation in the model. On the other hand, orthogonal component is empirically important, however the posterior inference about the angle of coordinate axes in (2) is extremely sensitive with respect to the value of correlation parameter $\rho$.

Also, restrictions in generalised model $M_{\rho, \varphi}$ affect noticeably inference about shape parameters, i.e., $\eta^{\prime} s$ and $v^{\prime} s$. According to results presented in Table III the DAX30 returns (included in our bivariate setting as a second variable) suggest the shape of distribution closer to the Student- $t$ case as the posterior distributions of $v_{2 \mathrm{~L}}$ and $v_{2 \mathrm{R}}$ are localised closer to the value $v=2$ than in case of FTSE100 returns. This effect seems qualitatively invariant with respect to the choice of the method of calculation of daily returns, i.e., in SERIES I and SERIES II. The shape of FTSE100 returns is closer to the Laplace case, as posterior distribution

\section{DATASET I}
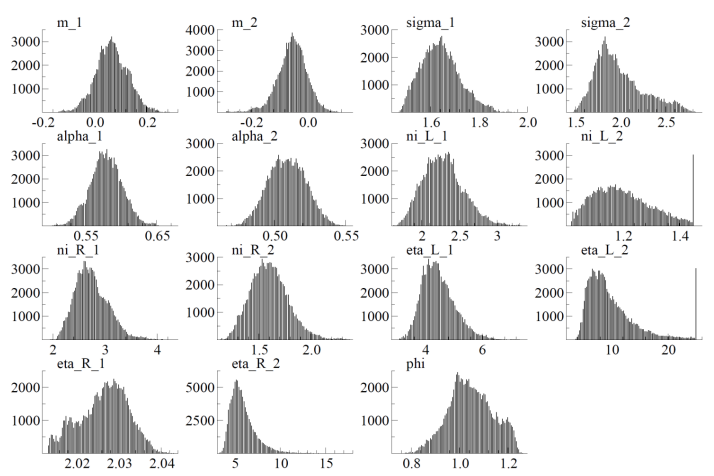

DATASET II
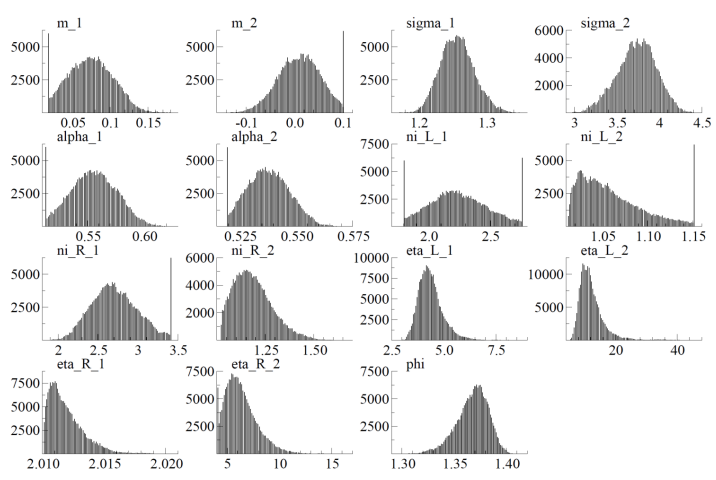

Fig. 9. Histograms of the marginal posterior distributions of parameters obtained in case of restricted model $M_{0, \varphi}$ with $\rho=0$ and $\varphi \in\left(-\frac{\pi}{2}, \frac{\pi}{2}\right) \approx$ $(-1.57,1.57)$.

of $v_{1 \mathrm{~L}}$ and $v_{1 \mathrm{R}}$ are localised closer to the value $v=1$. Another feature strongly supported by both datasets in case of model $M_{\rho, \varphi}$ is tail asymmetry of the distribution of FTSE100 returns and qualitatively the same left and right tail for the distribution of DAX returns.

Analysing the posterior inference about shape parameters in case of restricted models $M_{0, \varphi}$ and $M_{\rho, 0}$ again we report substantial sensitivity of results with respect to restrictions $\rho=0$ or $\varphi=0$. Since both restrictions are not supported by the datasets, our analyses show that neglecting both correlation and orthogonal component may influence strongly results of inference about the shape and tail behaviour of the distribution of analysed series. Strong variability of posterior means of $\eta^{\prime} s$ and $v^{\prime} s$ in case of models $M_{0, \varphi}$ and $M_{\rho, 0}$ clearly indicate, that these misspecified models may lead to very different conclusions about the nature of dependence between analysed returns or misleading risk assessment.

The estimation uncertainty is fully taken into account in the posterior-predictive distribution, which is a mixture of sampling-theory predictive density with mixing distribution being the joint posterior. Hence the estimation uncertainty is 'integrated out'. Iso-densities of the distribution are depicted in Fig. 11. 


\section{DATASET I}
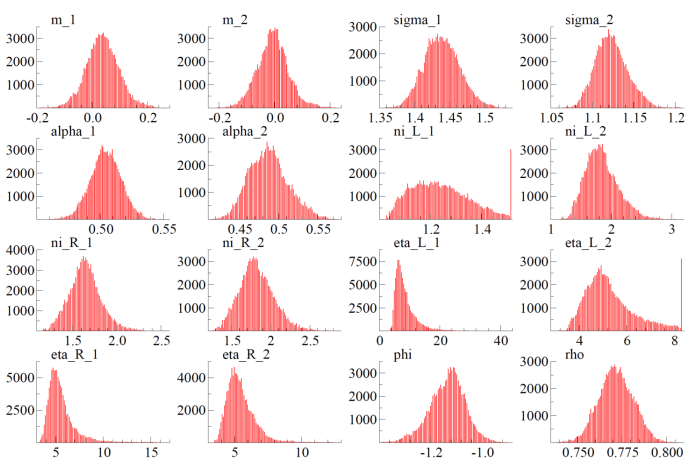

DATASET II
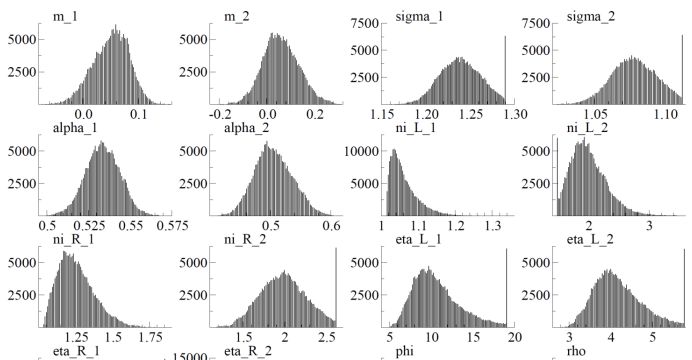

$2500-$
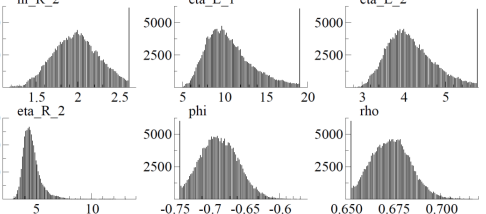

Fig. 10. Histograms of the marginal posterior distributions of parameters obtained in case of unrestricted model $M_{\rho, \varphi}$ i.e. with with $\rho \in(-1,1)$ and $\varphi \in\left(-\frac{\pi}{2}, \frac{\pi}{2}\right) \approx(-1.57,1.57)$.
SERIES I
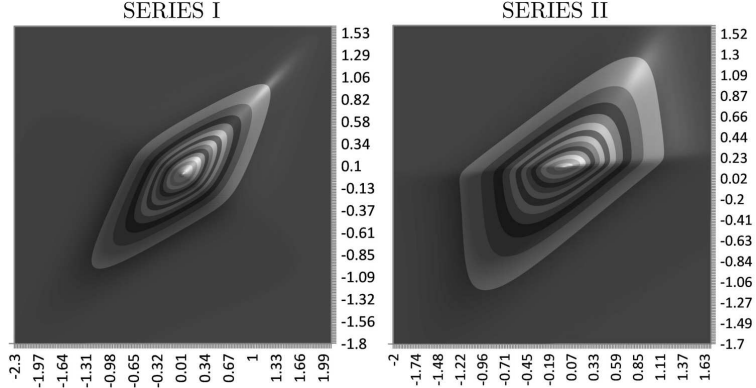

Fig. 11. Estimated density function of the posterior predictive distribution in case of unrestricted model $M_{\rho, \varphi}$.

Again, the densities do not resemble standard distributions nor the usual construct obtained as unconditional distributions from the standard dynamic models. The posterior predictive distribution analysed here provides a natural benchmark to long-term density forecasts from dynamic models and hence can be used to provide some sort of "boundary" values of risk measures.

\section{Conclusions}

We develop a class of flexible multivariate distributions that differs from the usual ones in two particular aspects. Firstly, we allow for high degree of stochastic heterogeneity across variables (addressing asymmetry and tail thickness issues), allowing for 5 shape parameters per dimension. Secondly, we introduce the dependence not only via covariances but also via rotations (which is possible due to generality of the distribution). This approach differs from the alternative ones, e.g., using the copula functions: here the form of marginal distribution is not directly controlled. However, the dependence structure is imposed by a well-defined transformation that goes beyond considering covariances only while it remains tractable also in higher dimensions. Hence, we provide a practical generalization of a product measure which allows for high degree of heterogeneity, more complicated dependence while avoiding some of the potential problems that arise within high-dimensional modelling using copula functions. The number of shape parameters increases linearly with dimension, but one could of course consider less-heavily parametrized special cases obtained by linear constraints. Therefore, the model provides a general framework allowing for the search for empirically relevant (restricted) special cases. Importantly, the construct considered here could be used to define conditional distribution in a dynamic model, which will be subject to further research.

\section{Acknowledgments}

This research was supported by the research grant 2017/25/B/HS4/02529 financed by the National Science Centre, Poland.

\section{References}

[1] M.G. Genton, Skew-Elliptical Distributions and Their Applications: A Journey Beyond Normality, Chapman \& Hall/CRC, Boca Raton (FL) 2004.

[2] F.Y. Edgeworth, Philos. Mag. 21, 308 (1886).

[3] K. Pearson, Nature 48, 615 (1893).

[4] K. Pearson, Nature 52, 317 (1895).

[5] E. De Forest, Analyst 9, 135 (1882).

[6] E. De Forest, Analyst 10, 1 (1883).

[7] F. Black, M. Scholes, J. Polit. Econ. 81, 637 (1973).

[8] R.C. Merton, Bell J. Econ. 4, 141 (1973).

[9] H.M. Markovitz, J. Finance 7, 77 (1952).

[10] W.B. Arthur, J.H. Hollan, B. LeBaron, R. Palmer, P. Tayler, Asset Pricing under Endogenous Expectations in an Artificial Stock Market, in: The Economy as an Evolving Complex System II Ed. W.B. Arthur, Vol. 27 of SFI Studies in the Sciences of Complexity, AddisonWesley, Redwood 1997. 
[11] P. Bak, M. Paczuski, M. Shibik, Physica A 246, 430 (1997).

[12] T. Lux, J. Econ. Behav. Organ. 33, 143 (1998).

[13] R. Cont, J.P. Bouchaud, Macroecon. Dyn. 4, 170 (2000).

[14] P. Saikkonen, M. Lanne, "A Skewed GARCH-in-Mean Model: An Application to U.S. Stock Returns", 2004, unpublished manuscript.

[15] P. Theodossiou, C.S. Savva, Manage. Sci. 62(6), 1598 (2015).

[16] C.S. Savva, P. Theodossiou, J. Financ. Econom. 16, 486 (2018).

[17] T.H. Kim, H. White, Finance Res. Lett. 1, 56 (2004).

[18] P. Theodossiou, Manage. Sci. 44, 1650 (1998).

[19] P. Christoffersen, S. Heston, K. Jacobs, J. Econom. 131, 253 (2003).

[20] P. Theodossiou, A. Theodossiou, "Event Studies and Outlier Returns: Symptoms, Consequences and Treatment" SSRN Electronic Journal (2019).

[21] P. Theodossiou, Multinational Finance J. 19, 223 (2015).

[22] P. Theodossiou, D. Tsouknidis, C.S. Savva, J. Royal Statist. Soc. Ser. A (2020).

[23] C.R. Harvey, A. Siddique, J. Finance 55, 1263 (2000).

[24] Ch. Adcock, Capital Asset Pricing for UK Stocks under the Multivariate Skew-Normal Distribution in: SkewElliptical Distributions and Their Applications: A Journey Beyond Normality Ed. M.G. Genton, Chapman \& Hall/CRC, Boca Raton (FL) 2004.
[25] A. Azzalini, A. Dalla Valle, Biometrika 83, 715 (1996).

[26] M. Pelagatti, S. Rondena, "Dynamic Conditional Correlation with Elliptical Distributions" Working Papers No. 20060508, 2006.

[27] L. Bauwens, S. Laurent, J. Bus. Econ. Stat. 23, 346 (2005).

[28] S.K. Sahu, D.K. Dey, M.D. Branco, Can. J. Stat. 31, 129 (2003).

[29] M. Pipien, "Orthogonal Transformation of Coordinates in Copula M-GARCH Models - Bayesian analysis for WIG20 spot and futures returns" NBP Working Papers 151, 2013.

[30] E.M. Iglesias, G.D.A. Phillips, Econom. Rev. 31, 532 (2012).

[31] A. Harvey, R.J. Lange, J. Time Ser. Anal. 38, 175 (2017).

[32] A. Azzalini, Scand. J. Stat. 12, 171 (1985).

[33] K.T. Fang, S. Kotz, K.W. Ng, Symmetric Multivariate and Related Distributions, Chapman and Hall, New York 1990.

[34] Zhu D., Zinde-Walsh V.,J. Econom. 148, 86 (2009).

[35] C. Fernández, M.F. Steel, J. Am. Stat. Assoc. 93, 359 (1998).

[36] D. Zhu, J.W. Galbraith, J. Econom. 157, 297 (2010).

[37] W.F. Sharpe, J. Finance 19, 425 (1964).

[38] R. Shiller, Am. Econ. Rev. 71, 421 (1981).

[39] M. Kalimipalli, R. Jha, EFA 2003 Annual Conference Paper No. 380, 2003. 